\section{A boost for high flyers}

\section{Gary Zank}

HIGH-ENERGY cosmic rays which bombard our atmosphere have been accelerated to their high velocities by collisions with irregular magnetic fields in our Galaxy, Bryant et al. show on page 582 of this issue ${ }^{1}$. With their sophisticated calculations, the authors show that the mechanism, first proposed by Fermi ${ }^{2}$ in 1949, can account for the remarkably high energies of the most energetic cosmic rays.

Thought at first to be $\gamma$-rays, cosmic rays are in fact composed predominantly of positively charged, highly energetic particles such as accelerated nuclei. The modern idea that cosmic rays are associated with supernova remnants (the turbulent debris of exploded stars) dates back to a brilliant paper by Baade and Zwicky ${ }^{3}$ in 1934, but the first compelling theoretical explanation was that advanced by Fermi. Fermi argued that particles are energized through head-on collisions with moving magnetic 'mirrors' in the interstellar medium, the energy gained being proportional to their initial energy. Fermi imagined these mirrors would be associated with slowly moving molecular clouds which traverse the Galaxy, but the rapidly expanding shock fronts of supernova remnants are now considered a more likely site. The particles' resulting energy $(E)$ distribution (assuming their escape from the acceleration region does not depend on their energy) should be a power law whose (negative) exponent is the ratio of the particle acceleration timescale and the escape timescale: $N \propto E^{-T_{\text {acc }} / T_{\text {esc }}}$

Fermi's arguments, and subsequent refinements, are both compelling and disturbing - compelling because of their simplicity and universality, but disturbing because the full underlying physical theory is embedded in the two parameters $T_{\text {acc }}$ and $T_{\text {esc }}$. To account for the observed cosmic-ray spectrum, it is necessary for $T_{\text {acc }} \simeq 1.7 T_{\text {esc }}$, although there is no good physical reason for this factor, nor even for why $T_{\text {acc }} / T_{\text {esc }}$ should be constant throughout the Galaxy. Fermi's argument is so general that it can almost be regarded as an interesting property of a certain kind of stochastic system independent of any particular theory.

Perhaps the most damning argument against Fermi's original formulation of the problem is that cosmic rays, being at most $10^{6}-10^{7}$ years old (according to dating by ${ }^{11} \mathrm{Be}$ isotopes), have to be accelerated to their enormous energies very rapidly. Indeed, because the characteristic acceleration time can be related to the speed $u\left(10-40 \mathrm{~km} \mathrm{~s}^{-1}\right.$, for Fermi's molecular clouds) with which the magnetic mirrors move through the interstellar medium $\left(T_{\mathrm{acc}} \propto(u / c)^{2}\right.$, where $c$ is the speed of light), there is simply not enough time for the mechanism to generate the most energetic cosmic rays.

Little further was done to improve cosmic ray I, GCRI). The particle acceleration time and short effective lifetime of supernova remnants $\left(10^{6}\right.$ years $)$, however, conspire to prevent particles gaining any energy above this cut-off. At the cut-off, something very curious happens to the spectrum (see figure). From $10^{13}$ to $10^{15} \mathrm{eV}$ there is a slight bump, or knee, which is followed by a second power-law region (GCRII, with differential intensity proportional to $E^{-3}$ ); at $10^{18} \mathrm{eV}$, the spectrum dips and then returns to an $E^{-2.7}$ distribution.

The high-energy dip and flattening is not particularly remarkable and is explained easily in terms of two independent sources producing power law spectra (perhaps a combination of galactic and extragalactic, EGCR, populations, although the intensities should be fairly similar). However, to obtain the continuous convex feature at the knee by combining two independent spectra would require something akin to a "cosmic conspiracy" (to use Jokipii's colourful description $^{5}$ ). It seems instead that the two spectra know of each Total energy (electronvolts) other and that the GCRII component is obtained as a result of further acceleration of the GCRI component. The smoothness of the fit indicates further that all GCRI particles in the vicinity of the supernova particleaccelerator cut-off energy must be allowed to participate in the postacceleration process. The mechanism proposed by Bryant et al. ${ }^{1}$ could reaccelerate the most energetic particles produced in supernova remnants up to energies of $10^{18}-10^{20} \mathrm{eV}$, if there is a suitable distribution of scattering centres with large magnetic fields in the Galaxy.

Development beyond this level of understanding will require the direct determination of cosmic-ray composition and spectra up to about $10^{16} \mathrm{eV}$, which colleagues and $I$ are now attempting ${ }^{6}$. If composition measurements show a gradual enrichment in heavy nuclei, then this might well be the signature of a multi-stage shock-acceleration process. On the other hand, an abrupt change in composition would signal the necessity for an entirely independent source and mechanism.

Gary Zank is at the Bartol Research Institute, University of Delaware, Newark, Delaware 19716, USA.

1. Bryant, D. A., Powell, G. I. \& Perry, C. H. Nature 356 582-583 (1992)

2. Fermi, E. Phys. Rev. 75, 1169-1174 (1949),

3. Baade, W. \& Zwicky, F. Proc. Natn. Acad. Sci. U.S.A. 20 239 (1934).

4. Axford, W. I. in Astrophysical Aspects of the Most Energetic Cosmic Rays (eds Nagano, M. \& Takahara, F.) 406-420 (World Scientific, Singapore, 1990)

作 process awaits further elucidation, the theory is generally satisfactory and reproduces the observed spectrum, proportional to $E^{-2.7}$, very well up to $10^{13} \mathrm{eV}$ (Axford terms ${ }^{4}$ this component galactic (eds Zank, G. P. \& Gaisser, T. K.) (American Institute of Physics, New York, in the press).

6. Gaisser, T. K., Stanev, T. \& Zank, G. P. Astrophys. J. (submitted).
5. Jokipii, J. R. in Particle Acceleration in Cosmic Plasmas 